\title{
DESAFIOS DA SEGURANÇA DO PACIENTE E A QUALIDADE EM SERVIÇOS DE ONCOLOGIA
}

Patrícia Peres de Oliveira - Enfermeira. Doutora em Educação. Mestre em Gerontologia pela PUC/SP. Docente da Graduação e do Programa de Pós-graduação Mestrado Acadêmico em Enfermagem na Universidade Federal de São João del-Rei/MG.

A inquietação a respeito da qualidade na prestação dos cuidados nas organizações em saúde é bastante antiga, apresenta-se como exemplo a desbravadora Florence Nightingale, enfermeira inglesa, que instituiu o primeiro modelo de melhoria contínua da qualidade em saúde durante a Guerra da Criméia, fundamentando-se em dados estatísticos e apresentação desses subsídios em gráficos. No ano de 1854, implementou rígidos padrões sanitários e de cuidados para a época, promoveu a redução das taxas de mortalidade e, em 1863, apontou preocupações relacionada à segurança do paciente no livro intitulado "notes on hospitals" (1).

A preocupação com qualidade do cuidado e com a segurança do paciente nas instituições de saúde tem surgido em âmbito global, inclusive, a Organização Mundial de Saúde (OMS) vem demonstrando inquietação com a segurança do paciente e, consequentemente, a qualidade dos serviços de saúde ${ }^{(2)}$, tanto que, é considerada alta prioridade na agenda de políticas dos seus países membros desde o ano $2000^{(3)}$.

Nos documentos da OMS, intitulado patient safety: making health care safer, sugere-se, aos serviços de saúde, recomendações como a adesão às práticas baseadas em evidências científicas e a acreditação. 0 processo de acreditação consiste em uma metodologia de avaliação externa, de caráter voluntário em sua contratação, periódico e reservado (por uma autoridade acreditada) a fim de garantir a qualidade da assistência por meio de padrões previamente definidos e demonstração de competência para realizar as atividades com segurança(4).

No Brasil, em 2013, o Ministério da Saúde instituiu o Programa Nacional de Segurança do Paciente (PNSP), por meio da Portaria número 529, com o objetivo geral de contribuir para a qualificação do cuidado em saúde, em todos os estabelecimentos de saúde do território nacional ${ }^{(5)}$. Ressalta-se que no artigo quarto do PNSP, os serviços de saúde devem constituir o Núcleo de Segurança do Paciente (NSP) e, a etapa da identificação dos riscos para a segurança do paciente, inclui a identificação das fontes (formais e informais) e a avaliação em processo de acreditação, em que resultados de pesquisa sobre qualidade e satisfação do usuário, entre outros, são primordiais. Todavia, atualmente, menos de $5 \%$ das instituições de saúde brasileiras tem a acreditação máxima pelas companhias certificadoras, sendo destas, três Unidades de Assistência de Alta Complexidade em Oncologia (UNACON) e apenas seis Centros de Alta Complexidade em Oncologia (CACON). 
A Agência Nacional de Saúde Suplementar, reguladora do mercado de planos privados de saúde brasileira, vinculada ao Ministério da Saúde, no ano de 2016, estabeleceu indicadores de qualidade para remuneração de profissionais e instituições de saúde e três linhas de cuidado prioritárias: oncologia, odontologia e assistência ao idoso. Para a oncologia, as determinações estão alicerçadas no diagnóstico precoce com estímulo a ações de promoção, prevenção e realização de busca ativa, continuidade entre o diagnóstico e o tratamento adequado em tempo cabível. Os resultados desejados são a melhoria na qualidade da atenção oncológica, baseados nas melhores práticas e em protocolos baseados em evidências científicas $^{(6)}$.

Atualmente, os desafios para a implementação de ações para melhoria da segurança do paciente e a qualidade em organizações oncológicas estão alicerçadas, principalmente, na necessidade de implementação de estratégias para evitar erros e eventos adversos na administração de antineoplásicos, a partir de protocolos institucionais para a padronização das condutas, criação de equipe multidisciplinar para a prevenção e avaliação de falhas no processo. São considerados relevantes indicadores no processo de avaliação de serviços de oncologia: incidência de queda de paciente; de não conformidade relacionada à administração de medicamentos como extravasamento de antineoplásicos; incidência de flebite; incidência de derramamento de quimioterápico; horas do enfermeiro/cuidado (mínimo, intermediário, semi-intensivo, intensivo); taxa de acidente de trabalho de profissionais de enfermagem; e taxa de rotatividade dos profissionais de enfermagem (turnover).

A prática da oncologia é interdisciplinar e os tratamentos geralmente possuem intervalos terapêuticos estreitos e complexos, propensos a comportamentos inesperados que podem resultar em danos substanciais ao paciente.

Além disso, reconhecendo que não podemos eliminar todos os riscos, enfatiza-se a atenção à segurança entre os funcionários das instituições de saúde, a fim de promover ainda mais a segurança e qualidade ao serviço. As situações que predispõem a redução da qualidade e aumento do risco de eventos adversos incluem o avanço tecnológico com insuficiente educação em serviço, falha na aplicação do processo de enfermagem, desmotivação, delegação da assistência sem supervisão apropriada e sobrecarga de trabalho(3).

$\mathrm{Na}$ oncologia clínica, a enfermagem tem importante relação com as práticas de quimioterapia antineoplásica. A fim de que tal agravo não ocorra, instituições com selo de acreditação máxima têm como norma, a dupla checagem da prescrição médica de protocolos quimioterápicos, sendo que somente após a validação do enfermeiro oncologista e do farmacêutico, ocorre a manipulação das drogas prescritas. Tratase de uma medida importante para evitar erro no cálculo de superfície corpórea do paciente, Outra estratégia relevante é o uso de sistemas de códigos de barra nas drogas manipuladas para cada paciente.

$\mathrm{Na}$ teleterapia, as novas tecnologias aumentaram a capacidade dos aceleradores lineares para fornecer mais radiação conforme os volumes tumorais e as metas planejadas, poupando o tecido saudável. No entanto, em casos raros, o aumento da complexidade pode resultar em acidentes fatais. Estudiosos 
apontam que a maioria dos acidentes nos setores de radioterapia está relacionado à documentação incompleta e/ou errada. Para evitá-los, as informações detalhadas necessitam estar descritas no plano de tratamento do paciente, as quais devem ser claramente comunicadas e documentadas por toda equipe (radioterapeutas, físicos, enfermeiros e oncologistas), além do desenvolvimento de software para aumentar a eficiência e eficácia do planejamento do tratamento de radioterápico ${ }^{(3)}$.

Para enfrentar todos os desafios da qualidade em serviços de oncologia, faz-se primordial garantir que, esses serviços sejam seguros e minimizem o risco de erro, encorajando a equipe a reportar incidentes, riscos e perdas próximas, bem como, basear as práticas sempre em evidências com protocolos institucionais. Outra estratégia é incentivar a participação do consumidor (envolvendo os pacientes/clientes e cuidadores) no cuidado, sendo que, as reclamações dos consumidores e os processos de feedback necessitam ser gerenciados em tempo hábil ${ }^{(1)}$.

Destarte, é importante criar nos serviços de saúde a noção do pensamento sistêmico, em que a responsabilização pela ocorrência de eventos adversos seja direcionada ao sistema de prestação de cuidados, a sua organização e funcionamento. Tal fato contribui para a construção de uma postura diferenciada frente ao dano, além da visão crítica de situações que venham causá-la. A análise dos aspectos relacionados ao cuidado favorece a melhoria no processo de trabalho de enfermagem, com consequente estabelecimento de uma assistência mais segura e de qualidade.

\section{Referências}

1. Ghahramanian A, Rezaei T, Abdullahzadeh F, Sheikhalipour Z, Dianat I. Quality of healthcare services and its relationship with patient safety culture and nurse-physician professional communication. Health Promot Perspect. 2017;7(3):168-74. Disponível em: https://www.ncbi.nlm.nih.gov/pubmed/28695106

2. Chera BS, Mazur L, Buchanan I, Kim HJ, Rockwell J, Milowsky MI, et al. Improving patient safety in clinical oncology: applying lessons from normal accident theory. JAMA Oncol. 2015;1(7):958-64. Disponível em: https://www.ncbi.nlm.nih.gov/pubmed/26182183

3. Schwappach DL, Gehring K. Frequency of and predictors for withholding patient safety concerns among oncology staff: a survey study. Eur J Cancer Care (Engl). 2015 May;24(3):395-403. Disponível em: https://www.ncbi.nlm.nih.gov/pubmed/25287114

4. World Health Organization (WHO). Patient Safety: Making health care safer. 2009. [página na Internet]. [acessado 2017 jun 20]. Disponível em: http://apps.who.int/iris/bitstream/10665/255507/1/WHO-HIS-SDS-2017.11-eng.pdf

5. Brasil. Portaria n. 529, de 1ำ de Abril de 2013: Institui o Programa Nacional de Segurança do Paciente (PNSP) [online]. Brasília (DF): Ministério da Saúde; 2013 [acesso 2017 Ago 18]. Disponível em: http://bvsms.saude.gov.br/bvs/saudelegis/gm/2013/prt0529 0104 2013.html 
6. Oliveira DF, Kornis GEM. A política de qualificação da saúde suplementar no Brasil: uma revisão crítica do índice de desempenho da saúde suplementar. Physis. 2017; 27(2): 207-31. Disponível em: https://www.researchgate.net/publication/318713561 A politica de qualificacao da saude suplementa $\underline{r}$ no Brasil uma revisao critica do indice de desempenho da saude suplementar

Como citar este artigo:

Oliveira PP. Desafios da Segurança do Paciente e a Qualidade em Serviços de Oncologia. Revista de Enfermagem do CentroOeste Mineiro. 2017;7:eEditorial. [Access 